Article

\title{
Single-Solution-Based Vortex Search Strategy for Optimal Design of Offshore and Onshore Natural Gas Liquefaction Processes
}

\author{
Muhammad Abdul Qyyum ${ }^{1,+}\left(\mathbb{D}\right.$, Muhammad Yasin ${ }^{2,+}$, Alam Nawaz ${ }^{1,+}{ }^{+}$, Tianbiao He ${ }^{3}$, \\ Wahid Ali ${ }^{4}{ }^{(D)}$ Junaid Haider ${ }^{1}$, Kinza Qadeer ${ }^{1}$, Abdul-Sattar Nizami ${ }^{5}$, \\ Konstantinos Moustakas ${ }^{6, *(D)}$ and Moonyong Lee ${ }^{1, *}$ \\ 1 School of Chemical Engineering, Yeungnam University, Gyeongsan 712-749, Korea; \\ maqyyum@gmail.com (M.A.Q.); alam.chem@gmail.com (A.N.); haiderjunaid92@gmail.com (J.H.); \\ kinzaqyyum@gmail.com (K.Q.) \\ 2 Bioenergy \& Environmental Sustainable Technology (BEST) Research Group, Department of Chemical \\ Engineering, COMSATS University Islamabad (CUI), Lahore Campus, Defense Road, Off Raiwind Road, \\ Lahore 54000, Pakistan; myasin@cuilahore.edu.pk \\ 3 Department of Gas Engineering, College of Pipeline and Civil Engineering, China University of \\ Petroleum (East China), Qingdao 266580, China; hetianbiao@upc.edu.cn \\ 4 Department of Chemical Engineering Technology, College of Applied Industrial Technology (CAIT), \\ Jazan University, Jazan 45971, Saudi Arabia; wahid8petro@gmail.com \\ 5 Sustainable Development Study Center, Government College University, Lahore 54000, Pakistan; \\ asnizami@gcu.edu.pk \\ 6 Unit of Environmental Science \& Technology, School of Chemical Engineering, National Technical University \\ of Athens, 15780 Athens, Greece \\ * Correspondence: konmoust@central.ntua.gr (K.M.); mynlee@yu.ac.kr (M.L.); Tel.: +30-2107723108 (K.M.); \\ +82-53-810-2512 (M.L.) \\ + These authors contributed equally.
}

Received: 6 March 2020; Accepted: 30 March 2020; Published: 5 April 2020

Abstract: Propane-Precooled Mixed Refrigerant (C3MR) and Single Mixed Refrigerant (SMR) processes are considered as optimal choices for onshore and offshore natural gas liquefaction, respectively. However, from thermodynamics point of view, these processes are still far away from their maximum achievable energy efficiency due to nonoptimal execution of the design variables. Therefore, Liquefied Natural Gas (LNG) production is considered as one of the energy-intensive cryogenic industries. In this context, this study examines a single-solution-based Vortex Search (VS) approach to find the optimal design variables corresponding to minimal energy consumption for LNG processes, i.e., C3MR and SMR. The LNG processes are simulated using Aspen Hysys and then linked with VS algorithm, which is coded in MATLAB. The results indicated that the SMR process is a potential process for offshore sites that can liquefy natural gas with $16.1 \%$ less energy consumption compared with the published base case. Whereas, for onshore LNG production, the energy consumption for the C3MR process is reduced up to $27.8 \%$ when compared with the previously published base case. The optimal designs of the SMR and C3MR processes are also found via distinctive well-established optimization approaches (i.e., genetic algorithm and particle swarm optimization) and their performance is compared with that of the VS methodology. The authors believe this work will greatly help the process engineers overcome the challenges relating to the energy efficiency of LNG industry, as well as other mixed refrigerant-based cryogenic processes.

Keywords: natural gas; single mixed refrigerant; propane-precooled mixed refrigerant; liquefaction process; energy efficiency; compression power 


\section{Introduction}

The continuous growth of human society will adversely affect the world energy requirements in the near future [1]. The estimates indicate an increase in the worldwide energy of up to $56 \%$ from 2010 to 2040 where India and China are the main contributors with increments of $112 \%$ in the same era [1]. Despite the intense research on renewable resources, it is projected that $76 \%$ of energy needs will be met by fossil fuels until 2040 [2]. Among conventional energy resources (i.e., gas, oil, and coal), Natural Gas (NG) has become essential because of its low Greenhouse Gas (GHG) emissions and increased thermal efficiency [3]. NG is the cleanest fuel with least contribution to climate change and air pollution $[4,5]$. As a result, a significant shift from coal to NG has been witnessed worldwide $[6,7]$.

Most of the NG reservoirs are located in the remote areas, thus NG is transported to markets in gaseous or liquid (LNG) forms. The economical and easy transportation of NG over long distances $(>2000 \mathrm{~km})$ is a critical factor, which is solved through liquefaction approach. The transportation of NG as LNG is preferred because of its lower cost and high safety compared with a pipeline (gaseous) transportation over long distances [8]. Due to this, the pipeline gas trade has been reduced by seven times than the LNG trade [9]. However, a primary issue with LNG processes is operating expenditure in terms of energy required to liquefy NG. It exhibits low energy efficiency, and the energy required is related to the compression power (shaft work) for refrigeration to liquefy NG. The shaft work for refrigeration cycles is dependent on the flow rates of the ingredients of mixed refrigerant (MR) and cycle operating conditions (e.g., refrigerant evaporation and condensation pressure) [10]. The optimal execution (i.e., via determining the optimal operating pressures and composition of the mixed refrigerant) of the liquefaction processes is minimized by the overall compression power [11,12]. This eventually enhances process efficiency by exploiting economic advantages with low energy requirements. Hence, several studies have improved liquefaction processes via sole optimization. For example, Lee et al. [13] determined the optimal design of the SMR case with minimum shaft work. Shah et al. [14] were the first to study multi-objective optimization of the C3MR process. They optimized the C3MR process by considering the total shaft work requirement, capital cost, total annual cost, and total hydrocarbon inventory as objective functions.

The process optimization is developed through adaptation of commercial process simulators, and this exhibits comprehensive thermodynamic libraries. The LNG processes are simulated in commercial simulators and optimized by developing a connection between both software environments. For instance, Aspelund et al. [15] built a simulation environment linked with the optimization algorithm. They used Aspen Hysys ${ }^{\circledR}$ to model SMR process and subsequently optimized it using a hybrid strategy by combining Nelder-Mead Downhill Simplex and Tabu search algorithms. Khan et al. [16] decreased the overall compression energy requirement of the SMR process using Non-Linear Programming (NLP) and particle swarm paradigm. Wang et al. [17] designed the C3MR process in Aspen Plus ${ }^{\circledR}$ and presented an optimal design through Sequential Quadratic Programming (SQP). Hatcher et al. [18], Lee et al. [19], and Mortazavi et al. [20] also modeled the C3MR process and subsequently optimized it via the Box method, Successive Reduced Quadratic Programming (SRQP), and hybrid optimization (i.e., Genetic Algorithm (GA) and SQP). Lee et al. [21] applied multi-objective optimization (using SQP) via gProms process simulator to find an optimal design of SMR process. Furthermore, Lee and Moon [22] have also used the SRQP to find optimal designs of SMR and C3MR liquefaction processes. They applied a mathematical optimization model that includes the cost model, as well as thermodynamic model. However, Tsay and Baldea [23] reported that modeling, simulation, and optimization become more complicated when phase transition and recycle streams are incorporated into refrigeration cycles. Therefore, they used equation-oriented modeling and optimization strategies $[24,25]$ to find the optimal design of SMR liquefaction processes. Moreover, Vikse et al. [26] presented a versatile simulation method to find an optimal design of complex SMR process. They investigated the capability of nonsmooth framework (i.e., equation-oriented modeling) for the optimal design of SMR process. Ali et al. [27] employed Generalized Polynomial Chaos (gPC) based surrogate modeling approach to study the operational reliability of the SMR process. Most recently, Zhu et al. [28] performed 
experimental investigation to analyze the flow distribution in plate-fin heat exchanger that is mostly used in the SMR process. Khan et al. [11] proposed a mixed refrigerant composition selection method based on the boiling point difference and specific refrigeration effect of individual components in mixed refrigerant. The proposed selection criteria were applied to the MR used in SMR and C3MR system; and a decrease in energy consumption for compression was observed. In another similar study, $\mathrm{Xu}$ et al. [29] developed a correlation between the ambient temperature and mixed refrigerant composition to evaluate energy utilization for PRICO LNG process. Qadeer et al. [30] adopted krill-herd optimization strategy for optimal solution of process conditions and MR composition of the SMR process.

Recently, a single-solution based Vortex Search (VS) [31] algorithm has been evaluated for the design optimization of complex processes such as modified SMR [32] and self-recuperative high temperature co-electrolysis-based methanol production [33]. Authors have found best optimal designs through the VS approach. Previously [32], the VS algorithm was used for the design optimization of modifed SMR process (MR consists of five components; nitrogen, methane, ethane, propane, and i-pentane) instead of conventional and well known SMR process that uses four components, i.e., nitrogen, methane, ethane, and propane. Since the optimization of LNG processes exhibited a significant reduction in exergy destruction and an enhanced the energy efficiency. The process engineers always focus on optimizing the plant design and operation to increase the profits of the chemical processes by improving the energy efficiency (reducing the operating costs). However, the energy efficiency enhancement for the LNG plants through exclusive optimization is a challenging task by virtue of highly complex and nonlinear thermodynamic interactions between the constrained design variables and energy efficiency. Therefore, efficient and rigorous optimization of the LNG processes continues to be an ongoing issue.

The major contribution of this study is the implementation and evaluation of the single-solution based Vortex Search (VS) [31] methodology to find the optimal design variables (corresponding to minimal energy consumption) of the SMR (for offshore applications) and C3MR (for onshore applications) processes. The SMR and C3MR processes are simulated in Aspen Hysys ${ }^{\circledR}$ v9. The VS algorithm is coded and modified in MATLAB version 2018b. The simulated LNG processes are linked with VS through ActiveX functionality. Optimal findings are analyzed and compared with the well-known algorithms including the GA and Particle Swarm Optimization (PSO). The VS algorithm can be applied to find the optimal design of any complex chemical process by simulating in Aspen Hysys.

\section{Vortex Search Strategy}

The single-solution based VS algorithm was developed by Doğan and Ölmez [31]. The dominant characteristic of the VS algorithm is the adaptive interval (step) size phenomenon that significantly improves the functionality of the search mechanism. The VS algorithm program balances neighborhood weak locality and strong locality to determine the optimal solution. Furthermore, the algorithm almost converges at the optimum point, and thus, it reacts in an exploitative (strong locality) manner to adjust the updated solution towards the optimal result. Hence, the hunted radius decreases with the completion of each iteration. The VS algorithm deterministically locates a solution in the specified lower and upper bounds and converges to a global optimal point for the optimization. To examine the performance, the VS approach has been employed to solve the issue of the selection of an analog active filter component [34] and the optimal design of analog filter group delay [35].

In the Vortex Search Optimization (VSO) approach, the effectiveness and success of optimal solutions significantly depend on weak and strong locality. The weak and strong locality refers to small and large changes that occur in the current solution. A weak locality is required when the search procedure begins whereas strong locality is required when the optimization algorithm converges successfully close to the best optimal solution. Further, Figure 1 shows the optimization procedure using 
the VS algorithm. The major steps of the VS algorithm searching are; primary estimation (initialization of VS), candidate solutions, current solution substitution, and radius reduction methodology.

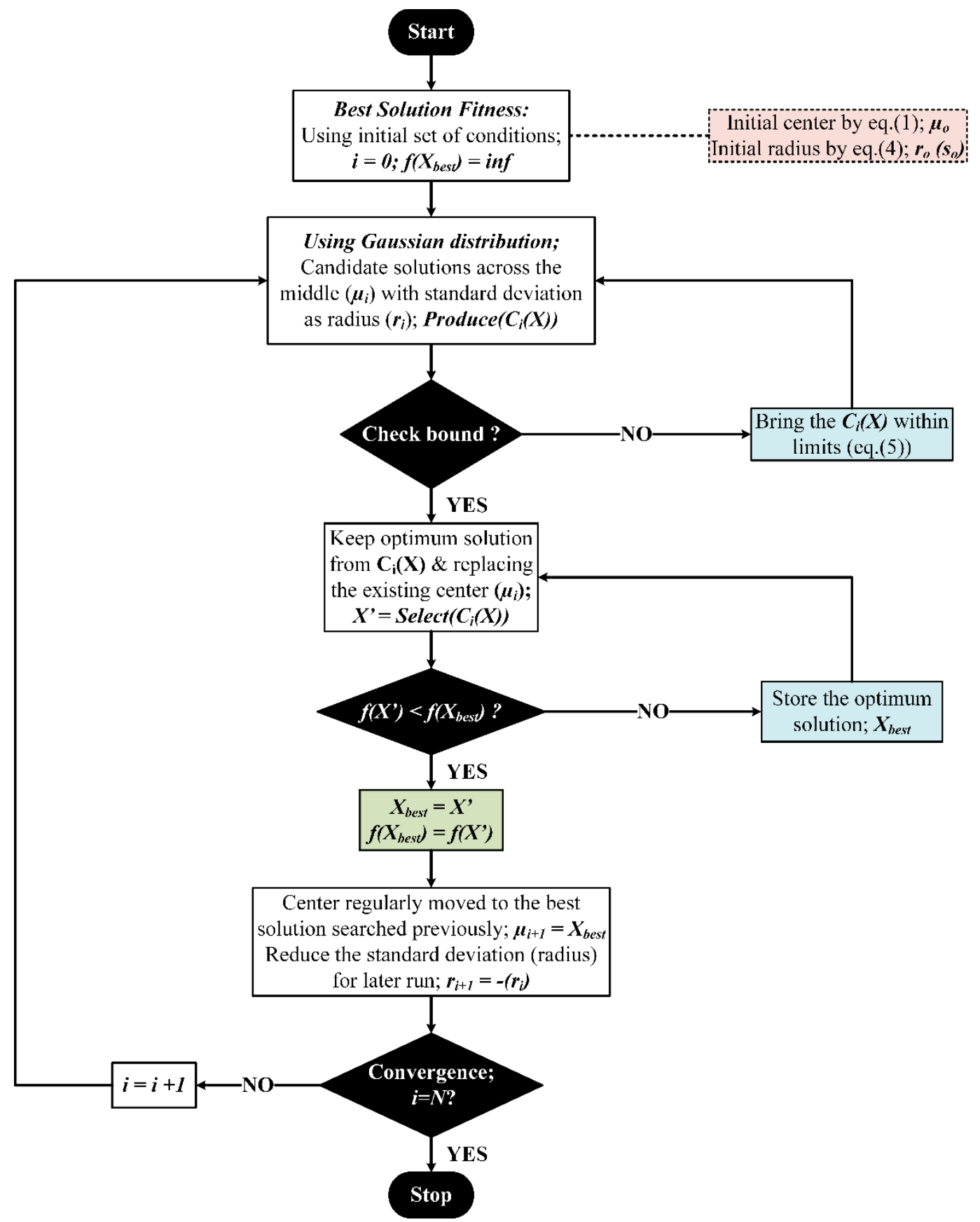

Figure 1. Searching flowchart for the vortex search (VS) algorithm.

\subsection{Primary Estimation}

The search strategy of the said algorithm is explained using a nested vortex pattern. Figure 2 shows a model of the VS algorithm in a bidimensional nested circle. The rivet of the search under the initial conditions is determined by the diameter of the outermost circle. The rivet or initial center $\left(\mu_{0}\right)$ is given by Equation (1) as follows:

$$
\mu_{0}=\frac{\text { upper bound }+ \text { lower bound }}{2}
$$




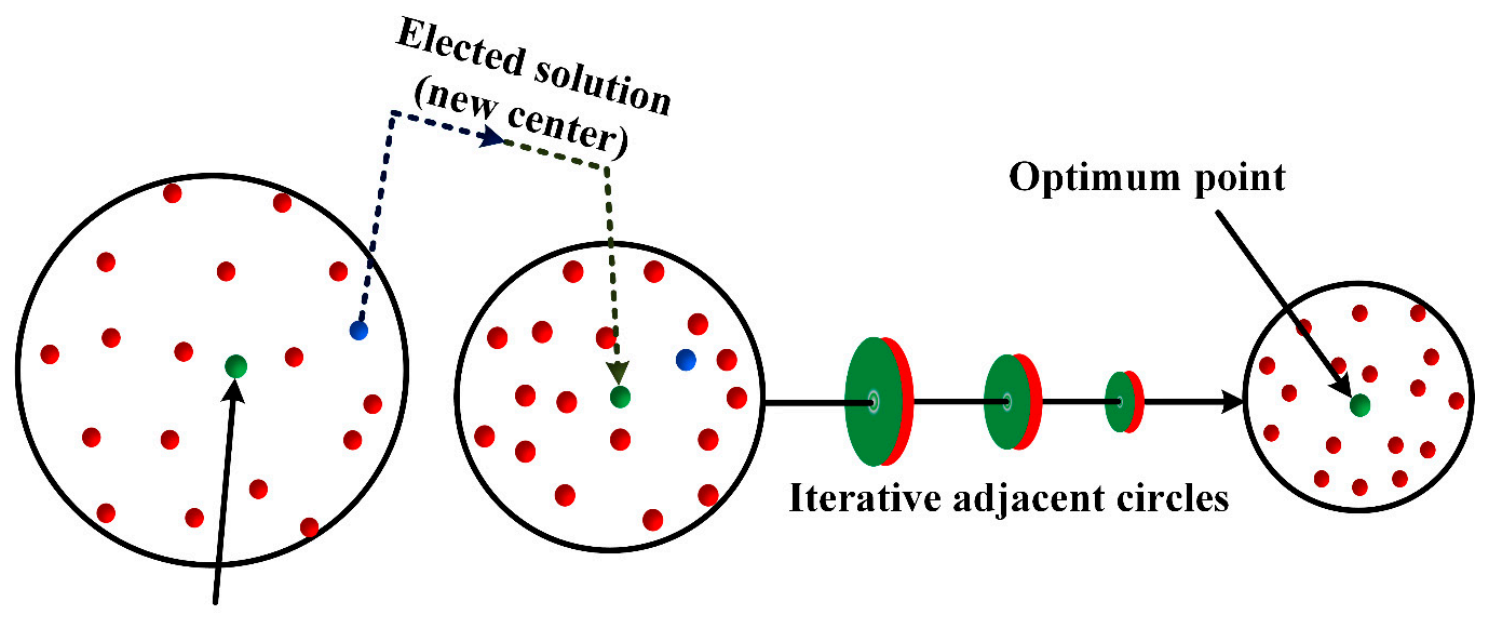

\section{Center at initial point}

Figure 2. Representation of the working search process using a model of the VS in a two-dimensional nested circle form.

\subsection{Candidate Solutions}

After evaluating the initial solution, a Gaussian distribution is used to calculate next neighbor solutions $C_{i}(X)=\left\{x_{1}, x_{2}, x_{3}, \ldots, x_{k}\right\}, k=1,2, \ldots, n$ as expressed in Equation (2) as follows:

$$
p(\zeta \mid \mu, v)=\frac{1}{\sqrt{(2 \pi)^{d}|v|}} \exp \left(-\frac{1}{2} \frac{(\zeta-\mu)^{T}(\zeta-\mu)}{v}\right)
$$

where $n$ denotes number of candidates' local optimal points, $i$ defines the number of count and $d$ represents the dimension number, and $\zeta$ and $\mu$ denote the vector for a haphazardly yielded variable and the sample mean (chosen as the center), respectively. Additionally, $v$ denotes the covariance matrix that is given by Equation (3) as follows:

$$
v=s^{2}[I], \text { dimesion }: \mathrm{d} \times \mathrm{d}
$$

where $s^{2}$ denotes the variance distribution and $I$ denotes the identity matrix. The standard deviation $\left(s_{0}\right)$ under the initial conditions is given by Equation (4) as follows:

$$
s_{0}\left(=r_{0}\right)=\frac{\max (\text { upper bound })-\min (\text { lower bound })}{2}
$$

where $s_{0}$ is considered as the initial radius $\left(r_{0}\right)$ and is differentiated to yield an entire covering of the weak vicinity in the neighborhood search area for a fully weak locality at the initial stage.

\subsection{Current Results Substitution}

The replacement phase of the nearest candidate result with the current solution commences with the solution $X^{\prime} \in C_{0}(X),(i=0)$ from $C_{0}(X)$, wherein the current circle center $\mu_{0}$ lies within the search space limits. If the new solutions are beyond the search space boundaries, then the candidate solutions are shifted into the specified bounds as given in Equation (5) as follows:

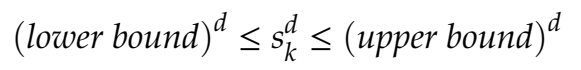

where $k$ varies from 1 to $n$, and $d$ symbolizes the dimension of bound limits. In the ensuant iteration, the obtained optimum solution $X^{\prime}$ denotes the center of the next circle. In the second step of the coevals phase, the active radius $\left(r_{1}\right)$ of the circle decreases, and a new set of vectors $C_{1}(X)$ is obtained across 
the new center. In the second step of the selection phase, the new solution set $C_{1}(X)$ is evaluated via $X^{\prime} \in C_{1}(X)$. If the selected solution advances to the old solutions, then it is saved.

Similarly, the assigned center in the third step is hypothetically saved as the new advanced/optimum solution to the point, as shown in Figure 3. The phenomenon continues until the completion criterion is satisfied.

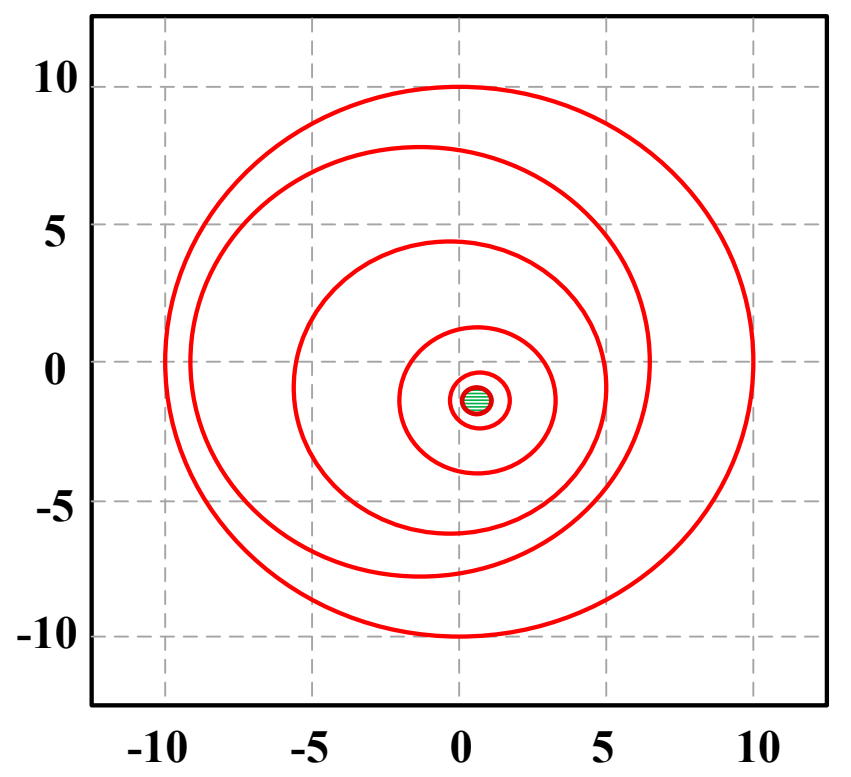

Figure 3. Vortex-pattern search space of the VS algorithm.

\subsection{Radius Reduction Methodology}

This is viewed as a type of reconciliation step-size improvement strategy that is employed in random seeking mechanisms to realize the potential benefits of the VS approach. The approach ensures the VS program to act in an exploratory (weak locality) fashion at the beginning and in the manner of a strong locality in the final measurements. To attain this type of a search mechanism, it is necessary to appropriately adjust the gain of the radius during optimization in each iteration. Therefore, the radius decrement process is described as an inverse incomplete gamma function. The VS algorithm is used to optimize a few benchmark objective functions to demonstrate the effectiveness of the algorithm, and the results are listed in Table 1. 
Table 1. Benchmark functions optimized using the VS algorithm.

\begin{tabular}{|c|c|c|c|c|}
\hline $\begin{array}{l}\text { Name of Function and } \\
\text { (Global Value) }\end{array}$ & Objective Function Equation & Minimum & Search Bound & Plot \\
\hline Schafer, (0) & $f(x)=0.5+\frac{\sin ^{2}\left(\sqrt{x_{1}^{2}+x_{2}^{2}}\right)-0.5}{\left(1+0.001\left(x_{1}^{2}+x_{2}^{2}\right)\right)^{2}}$ & 0 & {$[-100,100]$} & \\
\hline Goldstein-Price, (3) & $\begin{array}{c}f(x)=\left[1+\left(x_{1}+x_{2}+1\right)^{2}\left(19-14 x_{1}+3 x_{1}^{2}-14 x_{2}+6 x_{1} x_{2}+3 x_{2}^{2}\right)\right] \\
{\left[30+\left(2 x_{1}-3 x_{2}\right)^{2}\left(18-32 x_{1}+12 x_{1}^{2}+48 x_{2}-36 x_{1} x_{2}+27 x_{2}^{2}\right)\right]}\end{array}$ & 3 & {$[-2,2]$} & \\
\hline $\begin{array}{l}\text { Six Hump Camel Back, } \\
(-1.0316)\end{array}$ & $f(x)=4 x_{1}^{2}-2.1 x_{1}^{4}+\frac{1}{3} x_{1}^{6}+x_{1} x_{2}-4 x_{2}^{2}+4 x_{2}^{4}$ & -1.0316 & {$[-5,5]$} & \\
\hline Matyas, (0) & $f(x)=0.26\left(x_{1}^{2}+x_{2}^{2}\right)-0.48 x_{1} x_{2}$ & 0 & {$[-10,10]$} & \\
\hline
\end{tabular}




\section{Onshore and Offshore LNG Processes}

Many liquefaction technologies have been developed for onshore and offshore LNG production. The C3MR, DMR, and cascade processes are considered suitable candidates for onshore LNG production. Whereas, for offshore applications, the SMR, nitrogen-expander-based, and DMR processes are the promising options. The DMR process is considered for both onshore, as well as offshore applications [36]. Generally, the liquefaction process is selected based on NG reserve's location (onshore or offshore), production capacity, degree of complexity, and environmental conditions. However, considering the energy efficiency (operating costs), the SMR (PRICO ${ }^{\circledR}$ ) process is the most suitable candidate for small-scale and offshore applications compared with the expander-based LNG processes [37]. Although, for offshore applications, the nitrogen-expander-based liquefaction process have several dominant features such as safety and simplicity in operation but thermodynamically it is less attractive than the SMR process, due to high exergy destruction. Whereas, the C3MR process is considered as a promising candidate for onshore applications due to its relative high energy efficiency. Furthermore, this process is capable of producing $81 \%$ of the base-load LNG [38]. It is reported [39] that about $77 \%$ of the world's LNG plants are using the C3MR technology. Nevertheless, the process exhibits a high degree of complexity. Detailed process descriptions of the SMR and C3MR liquefaction processes are described in the forthcoming sections.

\subsection{SMR LNG Process Description}

A conventional SMR, alternatively known as the PRICO (poly refrigerant integrated cycle operations) process, was first introduced by Black and Veatch in 1970 [40]. Figure 4 shows that the SMR process consists of compressors to compress the MR in steps with intermediate coolers (air/water), i.e., a cryogenic multistream exchanger for exchanging heat between MR and feed natural gas and expansion valves (Joule-Thomson (JT)). In the SMR LNG cycle, a mixed refrigerant stream comprised of a range of mixture of methane, ethane, propane, and nitrogen is employed to produce a significant refrigeration effect to liquefy natural gas up to a temperature of approximately $-159{ }^{\circ} \mathrm{C}$ and a pressure of 1.3-2.0 bar. First, the SMR stream is introduced into a refrigeration cycle loop equipped with compression units, including after-coolers/inter-stage coolers. The MR gains heat during the compression process and is removed by air/water-cooled inter-stage coolers. Subsequently, the MR stream enters the cryogenic multistream exchanger and gets condensed. Then, the pressurized liquid MR passes through a JT valve where its pressure is lowered. At the outlet of this JT valve, the MR is obtained with liquid fraction $>0.85$. This MR (stream-5) exchanges the latent heat with incoming high-pressure MR (stream-14), as well as feed NG (stream-1) and is evaporated. Finally, it is exited as a superheated vapor (stream-6) from the LNG heat exchanger and is subsequently introduced into the compressor (K-1), thereby completing the refrigeration loop. 


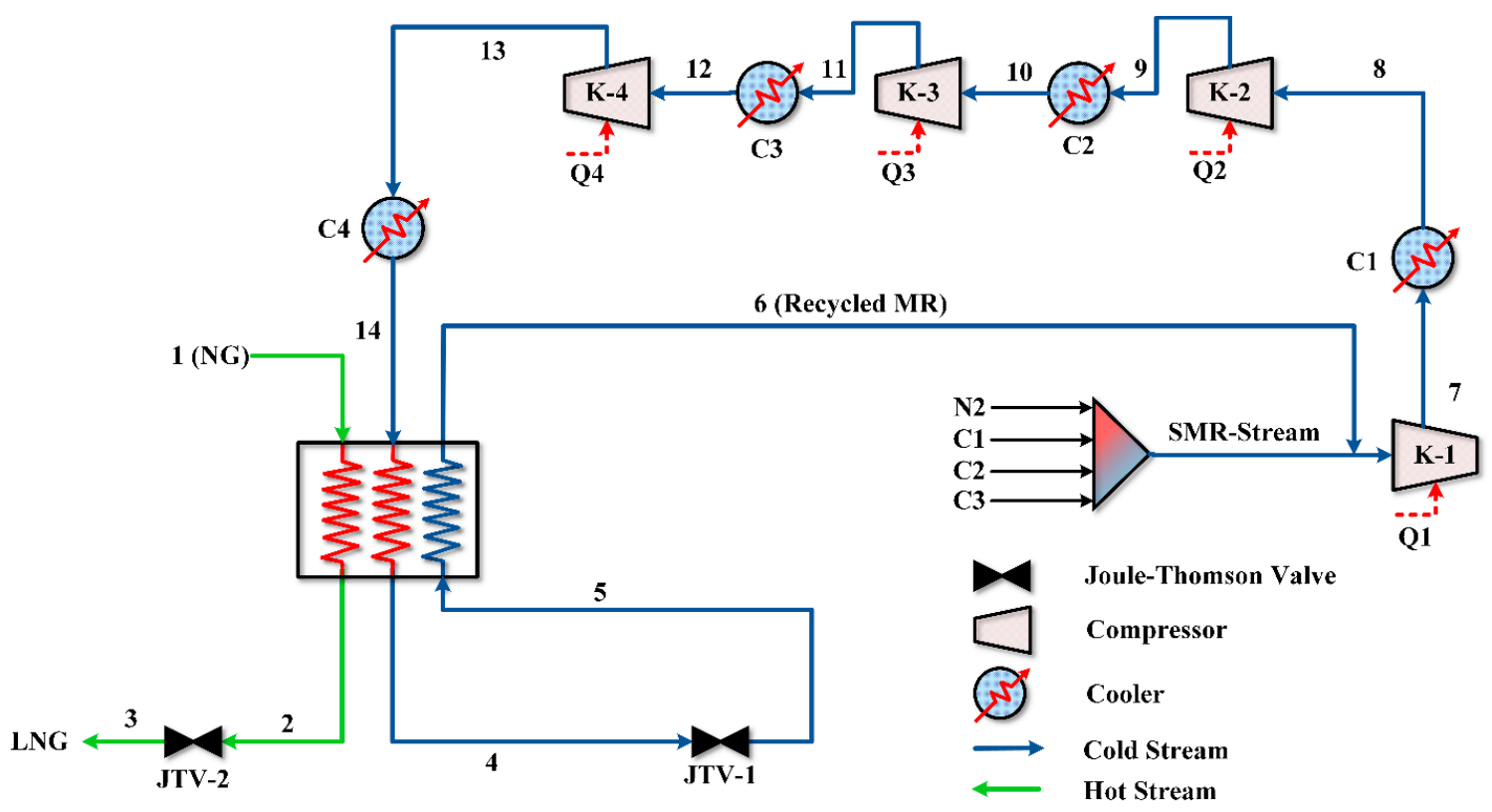

Figure 4. Process flow diagram of the single mixed refrigerant (SMR) process.

\subsection{C3MR LNG Process Description}

Although the SMR and C3MR exhibit resemblance in terms of the MR cycle, the degree of complexity of C3MR exceeds that of SMR. They utilize the same cryogenic heat exchanger (i.e., a plate and fin brazed with aluminum core) [11]. The C3MR process is less inviting for offshore applications due to its complex structure and large space requirements. Given its ability to match hot (natural gas) and cold (MR boiling) composite curves, the C3MR process performance is lower than that of other available LNG processes. The C3MR process uses the two refrigeration cycles; pure propane (C3) and MR-based refrigeration cycles as shown in Figure 5. The propane-based refrigeration loop is employed to precool the feed natural gas, as well as the mixed refrigerants. Subsequently, the process of liquefaction and subcooling of the feed natural gas stream is performed through the MR cycle.

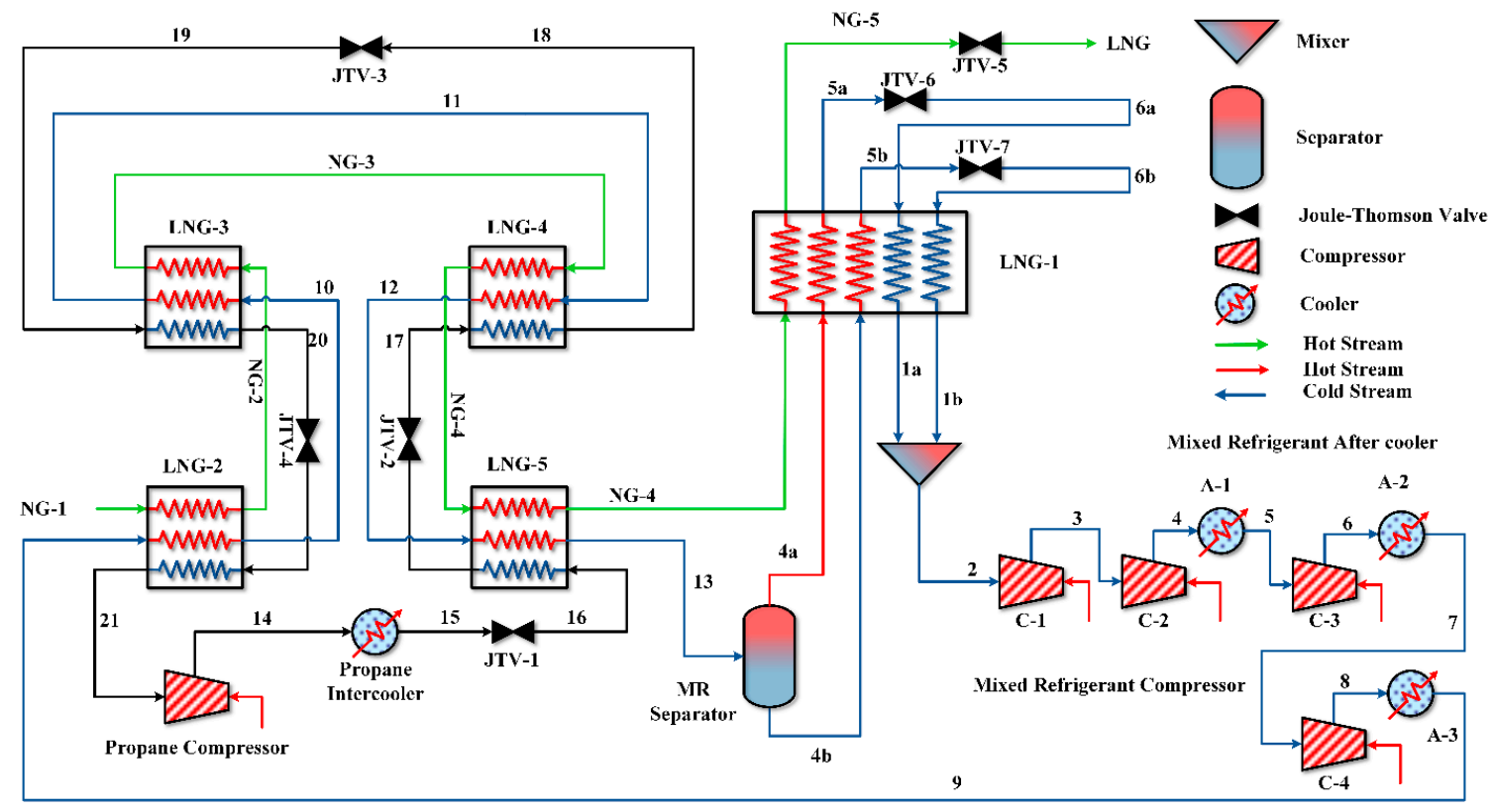

Figure 5. Process flow diagram of the propane-precooled mixed refrigerant (C3MR) process. 


\subsection{Simulation Basis for LNG Process Modeling}

In the study, Aspen Hysys is used to model the SMR and C3MR processes. Optimal conditions for the processes as determined by Khan et al. [32] are selected to create the base case study. Natural gas feed quality, other process conditions, and assumptions used in the simulation are highlighted in Table 2. Binary interactions parameters are predicted using the popular Peng-Robinson model, whereas the entropies and enthalpies are evaluated via the Lee-Kesler thermodynamic model. A Minimum Internal Temperature Approach (MITA) value is specified as $3{ }^{\circ} \mathrm{C}$ to obtain a more efficient design for the LNG multistream exchangers.

Table 2. Natural gas feed conditions and assumptions for Hysys modeling of the SMR and C3MR.

\begin{tabular}{cc}
\hline Property & Condition \\
\hline NG feed condition & \\
Flow rate & $1.0 \mathrm{~kg} / \mathrm{h}$ \\
Temperature & $32{ }^{\circ} \mathrm{C}$ \\
Pressure & $50 \mathrm{bar}$ \\
NG feed composition & Mole fraction \\
Methane & 0.9133 \\
Ethane & 0.0536 \\
Propane & 0.0214 \\
n-Butane & 0.0047 \\
i-Butane & 0.0046 \\
n-Pentane & 0.0001 \\
i-Pentane & 0.0001 \\
Nitrogen & 0.0022 \\
Intercooler outlet temperature & $40{ }^{\circ} \mathrm{C}$ \\
Vapor fraction boil-off-gas & $8.0 \%$ \\
Compressor isentropic efficiency & 0.75 \\
Fluid package & Peng-Robinson \\
Enthalpy/entropy calculation & Lee Kesler \\
Pressure drops across LNG cryogenic exchanger in SMR process \\
"Stream-1" to "Stream-2" & $1.0 \mathrm{bar}$ \\
"Stream-14" to "Stream-4" & $1.0 \mathrm{bar}$ \\
"Stream-5" to "Stream-6" & $0.1 \mathrm{bar}$ \\
Pressure drops across LNG exchanger in C3MR process & $1.0 \mathrm{bar}$ \\
"Stream-NG-1" to "Stream-NG-5" & $1.0 \mathrm{bar}$ \\
"Stream-4a" to "Stream-5a" & $0.5 \mathrm{bar}$ \\
"Stream-4b" to "Stream-5b" & $0.05 \mathrm{bar}$ \\
"Stream-6a" to "Stream-1a" & $0.05 \mathrm{bar}$ \\
"Stream-6b" to "Stream-1b" & negligible \\
\hline Heat loss to the environment in both SMR and C3MR processes &
\end{tabular}

\section{Optimization Problem Formulation for SMR and C3MR Processes}

The effectiveness of any optimization problem is examined by the mathematical problem formulation. The effectiveness significantly affects the type of optimal candidate set results and optimization elapse time. The following constitute the major components for any general optimization problem:

1. Target function(s).

2. Constraint function(s) and their limits(s).

3. Optimization variables.

4. Optimizing variable bounds (search area).

5. Other design parameters, if any.

Total energy requirement in terms of compression power for LNG processes is considered as a target/objective in several different design optimization studies. Therefore, the compression power 
required as an operating cost is considered as the objective function to liquefy unit mass flow of NG in both LNG processes. Hence, the mathematical optimization problem is expressed as follows:

$$
\operatorname{Minimize} f(Y)=\operatorname{Min} \cdot\left(\sum_{i=1}^{n} W_{i} / m_{L N G}\right)
$$

subject to the MITA values (inside all LNG heat exchangers) as constraints:

$$
\Delta T_{(\min )}(Y) \geq 3.0
$$

where $Y$ denotes the set of optimizing decision variables and $i$ denotes the number of compressors.

Parameters including the MR flow rate, condensation pressure, and evaporation pressure were selected as the design variables for SMR process. Whereas, for the C3MR process, the temperatures of the cooling stages in the propane cycle were also considered as the design variables in addition to the MR components flowrates, MR condensation, and evaporation pressures. Table 3 lists all decision variables (with their bounds) of the SMR and C3MR processes.

Table 3. Decision variables of the SMR and C3MR processes with their upper and lower bounds.

\begin{tabular}{ccc}
\hline Decision Variables & Lower Bound & Upper Bound \\
\hline Design $($ decision) variables & for SMR process & \\
High pressure of MR, $\mathrm{P}_{13}(\mathrm{bar})$ & 35.0 & 70.0 \\
Evaporation pressure, $\mathrm{P}_{5}(\mathrm{bar})$ & 1.1 & 4.0 \\
Flow rate of nitrogen, $m_{\mathrm{N} 2}(\mathrm{~kg} / \mathrm{h})$ & 0.1 & 0.65 \\
Flow rate of methane, $m_{\mathrm{C} 1}(\mathrm{~kg} / \mathrm{h})$ & 0.25 & 0.85 \\
Flow rate of ethane, $m_{\mathrm{C} 2}(\mathrm{~kg} / \mathrm{h})$ & 0.45 & 1.15 \\
Flow rate of propane, $m_{\mathrm{C} 3}(\mathrm{~kg} / \mathrm{h})$ & 2.0 & 3.5 \\
Design $($ decision $)$ variables for C3MR process & \\
MR cycle & & \\
High pressure of MR, $\mathrm{P}_{8}(\mathrm{bar})$ & 50.0 & 70.0 \\
Evaporation pressure, $\mathrm{P}_{6 \mathrm{a}}(\mathrm{bar})$ & 2.5 & 10.0 \\
Evaporation pressure, $\mathrm{P}_{6 \mathrm{~b}}(\mathrm{bar})$ & 2.5 & 10.0 \\
Flow rate of nitrogen, $m_{\mathrm{N} 2}(\mathrm{~kg} / \mathrm{h})$ & 0.05 & 0.45 \\
Flow rate of methane, $m_{\mathrm{C} 1}(\mathrm{~kg} / \mathrm{h})$ & 0.25 & 0.85 \\
Flow rate of ethane, $m_{\mathrm{C} 2}(\mathrm{~kg} / \mathrm{h})$ & 0.65 & 1.40 \\
Flow rate of propane, $m_{\mathrm{C} 3}(\mathrm{~kg} / \mathrm{h})$ & 0.15 & 0.8 \\
$\quad$ Propane cycle & \\
Propane 1st cooling stage $\left({ }^{\circ} \mathrm{C}\right)$ & 15.0 & 30.0 \\
Propane 2nd cooling stage $\left({ }^{\circ} \mathrm{C}\right)$ & 0.0 & 10.0 \\
Propane 3rd cooling stage $\left({ }^{\circ} \mathrm{C}\right)$ & -20.0 & -5.0 \\
\hline
\end{tabular}

Since the optimization of LNG processes is constrained by the MITA inside the LNG heat exchangers. Therefore, the overall compression power (i.e., objective function) was constrained by the MITA value of $3{ }^{\circ} \mathrm{C}$. The MITA value can be $1 \sim 3^{\circ} \mathrm{C}$. However, the MITA value of $3^{\circ} \mathrm{C}$ is more flexible to ensure the feasibility of the liquefaction with affordable economics.

\subsection{Constraint Handling Approach}

An exterior penalty function was used to handle the constraints (i.e., MITA value of $3.0^{\circ} \mathrm{C}$ ), and to further fold the constraints into overall compression power. It has been carried out by several LNG design optimization studies $[12,30,32,41]$.

Since the overall objective function is formed by combining the required power and constraint as specified in Equations (6) and (7) as follows: 
Finally, the objective function is reformulated in Equation (8) as follows:

$$
\operatorname{Minimize} P(Y)=\operatorname{Min}\left(\sum_{i=1}^{z} W_{i} / m_{L N G}+w(\max \{0,(3.0-\operatorname{MITA}(Y)\})\right.
$$

where $w$ denotes a positive penalty parameter, whose values correspond to 1 .

\subsection{Exergy Destruction Analysis}

Exergy for a refrigeration cycle is the minimum theoretical work required from the environment as the system comes from the dead state to the desired state. Exergy analysis identifies the energy flaws mainly due to the thermodynamic irreversibilities associated with each equipment, as well as the whole process. Thus, exergy analysis provides rigorous directions and attention for further improvement in order to reduce the exergy destruction as much as possible, which ultimately leads to enhance the overall process performance. The exergy destruction associated with SMR and C3MR processes for all cases (i.e., base case, GA-optimized, PSO-optimized, and VS-optimized) were performed using the expressions listed in Table 4 [42].

Table 4. Exergy destruction calculations expressions.

\begin{tabular}{cc}
\hline Equipment & Exergy Destruction $\mathbf{( k J} / \mathbf{h})$ \\
\hline Compressor & $E x_{\text {des }}=(\dot{\mathrm{m}})\left(E x_{\text {in }}-E x_{\text {out }}\right)-\dot{\mathrm{W}}$ \\
Interstage coolers & $E x_{\text {des }}=(\dot{\mathrm{m}})\left(E x_{\text {in }}-E x_{\text {out }}\right)$ \\
Multistream LNG heat exchanger & $E x_{\text {des }}=\sum(\dot{\mathrm{m}}) E x_{\text {in }}-\Sigma(\dot{\mathrm{m}}) E x_{\text {out }}$ \\
JT valves & $E x_{\text {des }}=(\dot{\mathrm{m}})\left(E x_{\text {in }}-E x_{\text {out }}\right)$ \\
\hline
\end{tabular}

\section{Results and Discussion}

The approach temperature (temperature difference) curve also known as TDCC provides information about the behavior of MITA value along the length of the LNG heat exchangers. Accordingly, the efficient heat transfer can be achieved when the height of the TDCC is close to the specified MITA value of $3{ }^{\circ} \mathrm{C}$. The performance of the LNG processes can also be observed through temperature-heat flow composite curves (THCC). The gap between THCC presents the exergy destruction associated with the LNG heat exchanger. Figure 6 shows the TDCC and THCC comparison of the VSO-optimized SMR process and the published base case. It can be observed that there is a larger gap between the THCC of base case and the VSO-optimized SMR; especially in a temperature range of -70 to $40^{\circ} \mathrm{C}$. This large gap indicates the exergy destruction inside the cryogenic LNG heat exchanger, which ultimately leads to low energy efficiency. It is necessary to reduce the space/gap between THCC to decrease exergy destruction. Thus, the MR flow should be at the optimal values to cope with the inefficiency present in the system and minimize the compression power required to liquefy the natural gas. Given the application of VS optimization approach, as clearly seen in Figure 6d, the triangular space is smaller than that of triangular area in Figure $6 \mathrm{~b}$. This reduction in the gap between THCC provides a significant energy savings compared with the base case of the SMR process. It is noted that high MITA value inside multistream heat exchanger continues to exist in the temperature range of -50 and $40^{\circ} \mathrm{C}$ (Figure 6d). 

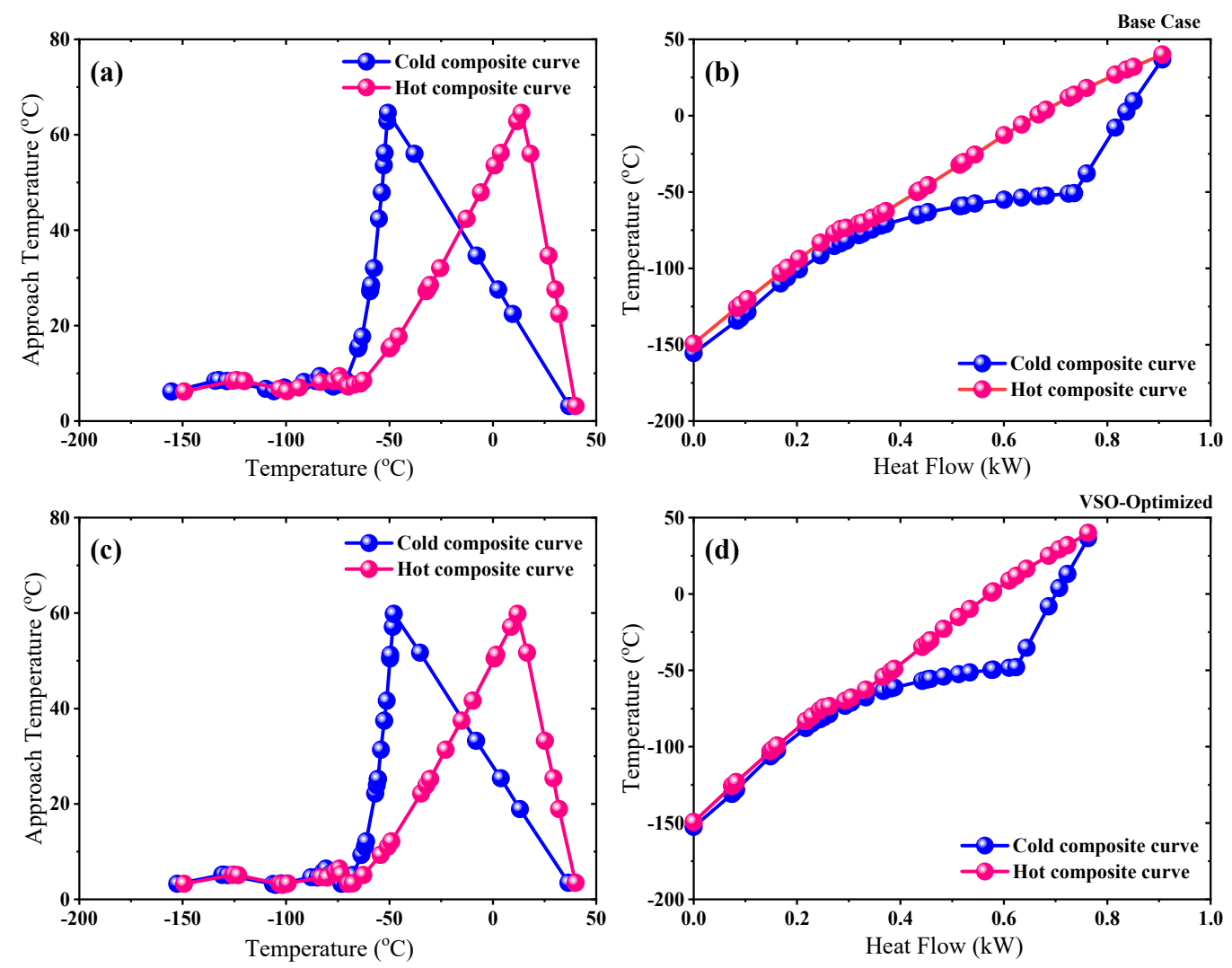

Figure 6. Base case (a) temperature difference composite curve (TDCC) and (b) temperature-heat flow composite curve (THCC) in comparison with the VS-optimized (c) TDCC and (d) THCC curves of the SMR process.

The high MITA value is principally due to the existence of the more volatile component, i.e., propane. The MITA value is reduced via precooling the feed gas and MR prior to entering the LNG cryogenic heat exchanger. This type of precooling conclusively enhances the operation of the C3MR plant by decreasing the temperature gradient in the LNG cryogenic exchanger through propane precooling.

Figure 7 shows the graphical results of the C3MR process for the VS-optimized and base case study. The VS-optimized C3MR process significantly reduces the enclosed space between the THCC as shown in Figure 7d, whereas the composite curves in the base case exhibit a relatively larger gap.

With respect to a feasible and reliable operation of any cryogenic plant, the composite curve matching approach is widely used as a thermodynamic graphical approach to determine the optimal performance of any heat exchange processes. To obtain high energy efficiency of a LNG process with low required compression power, the hot and cold steam temperature profile (i.e., composite curves) must be close to each other provided it follows the feasible approach temperature of $3^{\circ} \mathrm{C}$.

Table 5 also lists the temperature and pressure for all streams of VS-optimized SMR and C3MR processes. 

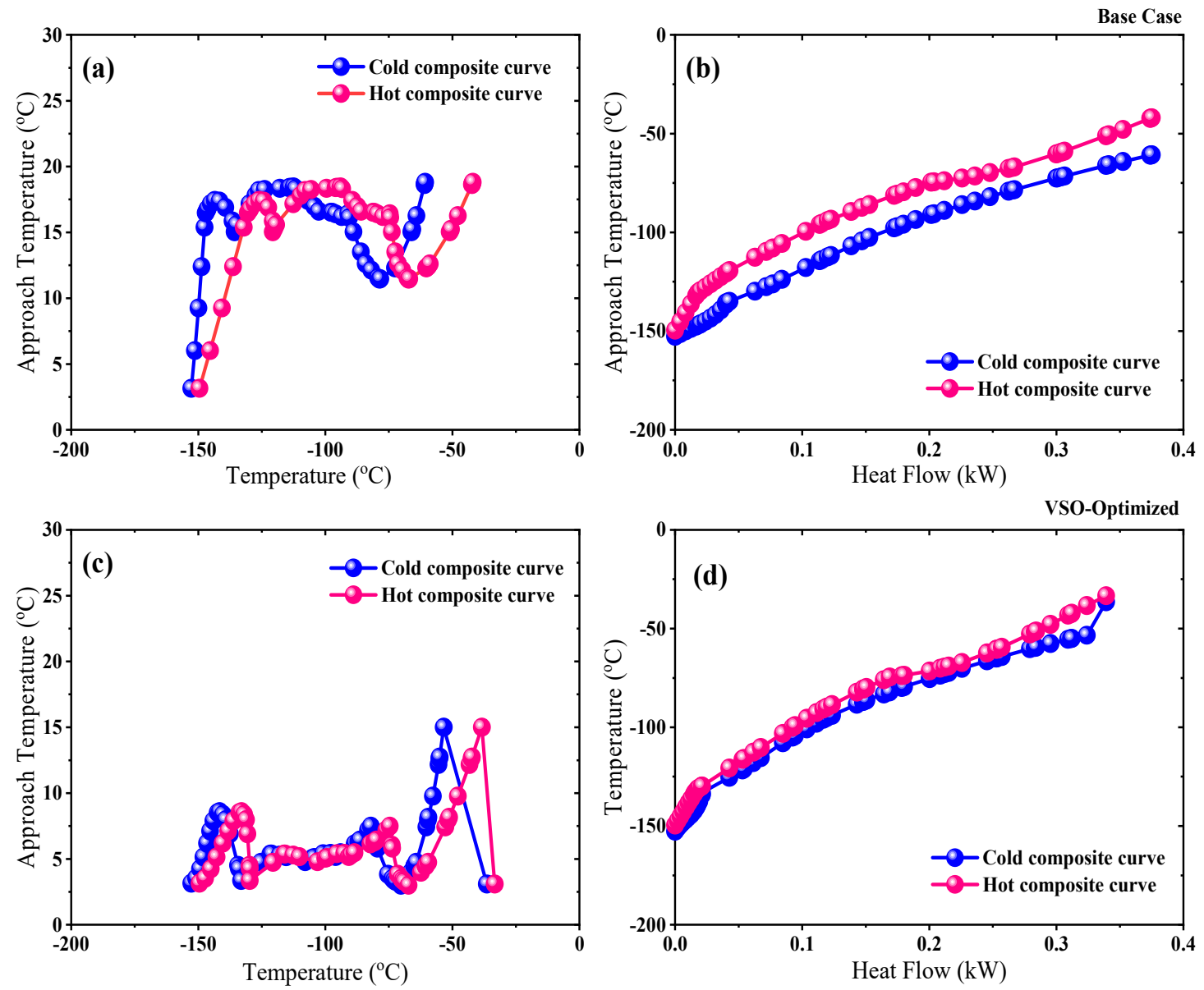

Figure 7. Base case (a) TDCC and (b) THCC curves in comparison with the VS-optimized (c) TDCC and (d) THCC curves of the C3MR process.

Table 5. Temperature and pressure for all streams of SMR and C3MR processes.

\begin{tabular}{cccccc}
\hline \multicolumn{3}{c}{ SMR Process } & \multicolumn{3}{c}{ C3MR Process } \\
\hline Stream & $\mathbf{T},\left({ }^{\circ} \mathbf{C}\right)$ & $\mathbf{P},($ bar) & Stream & T, $\left({ }^{\circ} \mathbf{C}\right)$ & P, (bar) \\
\hline 1 & 32.0 & 50.0 & $1 \mathrm{a}$ & -136.5 & 3.0 \\
2 & -149.3 & 49 & $1 \mathrm{~b}$ & -136.5 & 3.0 \\
3 & -158.5 & 1.209 & 2 & -56.4 & 3.0 \\
4 & -149.3 & 58.5 & 3 & -16.22 & 6.2 \\
5 & -152.6 & 1.65 & 9 & 40.0 & 55.0 \\
6 & 36.54 & 1.55 & $4 \mathrm{a}$ & -33.34 & 54.0 \\
7 & 90.39 & 3.858 & $4 \mathrm{~b}$ & -33.34 & 54.0 \\
8 & 40.0 & 3.858 & $5 \mathrm{a}$ & -119.4 & 53.99 \\
9 & 94.71 & 9.603 & $6 \mathrm{a}$ & -152.7 & 3.0 \\
10 & 40.0 & 9.603 & $5 \mathrm{~b}$ & -130.6 & 54.0 \\
11 & 96.53 & 23.9 & $6 \mathrm{~b}$ & -136.5 & 3.0 \\
12 & 40.0 & 23.9 & NG- 1 & 32.0 & 50.0 \\
13 & 101.5 & 59.5 & NG-4 & -33.34 & 49.0 \\
14 & 40.0 & 59.5 & NG-5 & -149.5 & 48.99 \\
SMR-stream & 36.54 & 1.55 & LNG & -158.5 & 1.209 \\
\hline
\end{tabular}

The optimization of both SMR and C3MR processes was performed by employing well-matured GA and PSO algorithms. The GA and PSO both are categorized as population-based algorithms. The performance of these algorithms strongly depends on the tuning parameters, as well as initial point generation. Table 6 lists the tuning parameters of PSO and GA, which were fixed during the 
optimization of SMR and C3MR. These parameters for GA and PSO were adopted from the recent LNG optimization studies [12,41,43].

Table 6. Particle swarm optimization (PSO) and genetic algorithm (GA) parameters used to set the optimization framework for SMR and C3MR processes.

\begin{tabular}{cccc}
\hline PSO & \multicolumn{2}{c}{ GA } \\
\hline Parameter & Value & Parameter & Value \\
\hline Number of particles & 30.0 & Number of population & 200.0 \\
Cognition learning parameter & 2.0 & Selection method & Stochastic uniform \\
Social learning parameter & 2.1 & Mutation & Adaptive feasible \\
Maximum velocity of particle & 4.0 & Crossover function & Scatter \\
Inertial weight & $0.9-0.2$ & Fraction of migration & 0.2 \\
& & Number of generations & 200.0 \\
\hline
\end{tabular}

Furthermore, Tables 7 and 8 list a comparison of the VS-optimized results of SMR and C3MR processes, respectively, with their previously published study results using other well-known optimization algorithms. The base cases for SMR and C3MR were taken from [44]. The SMR and C3MR processes consume 0.44 and $0.3602 \mathrm{~kW}$ compression power to liquefy $1 \mathrm{~kg}$ NG with $92 \%$ liquefaction rate, respectively.

In case of the SMR process optimization, the operational energy improvements in the compression power correspond to 0.4034 and $0.3862 \mathrm{~kW}$ via GA and PSO approaches, respectively. The GA optimized SMR process consumes $8.32 \%$ less energy when compared to that of the SMR base case, whereas the PSO-optimized SMR process exhibits $12.23 \%$ energy savings when compared with that of the SMR base case. Finally, the VS algorithm is used to optimize the SMR process, and this ensures top-rated optimal conditions over the present approaches. The total compression power decreases by up to $0.3691 \mathrm{~kW}$, and this corresponds to energy savings of $16.1 \%$ when compared to that of the base process. In the case of GA and PSO optimization of C3MR, the compression power decreases from 0.3602 to $0.2778 \mathrm{~kW}$ and $0.2754 \mathrm{kWh}$ with energy savings equivalent to $22.9 \%$ and $23.5 \%$, respectively, when compared to those of the base case. Finally, the VS algorithm results in a power saving of $0.26 \mathrm{kWh}$ in the compression mode having power savings of approximately $27.8 \%$ when compared to that of the base case.

Table 7. Summary and comparison of the optimization results of the VS-optimized SMR with optimized SMR process using other well-known optimization algorithms.

\begin{tabular}{ccccc}
\hline Decision Variables & Base Case [44] & GA Optimized & PSO Optimized & VS Optimized \\
\hline High pressure of $\mathrm{MR}, \mathrm{P}_{13}(\mathrm{bar})$ & 48.0 & 45.72 & 54.50 & 59.50 \\
Evaporation pressure of $\mathrm{MR}, \mathrm{P}_{5}(\mathrm{bar})$ & 1.30 & 1.680 & 2.10 & 1.550 \\
Flow rate of nitrogen, $m_{N 2}(\mathrm{~kg} / \mathrm{h})$ & 0.2690 & 0.3300 & 0.2200 & 0.1650 \\
Flow rate of methane, $m_{C 1}(\mathrm{~kg} / \mathrm{h})$ & 0.5290 & 0.4510 & 0.5900 & 0.4630 \\
Flow rate of ethane, $m_{C 2}(\mathrm{~kg} / \mathrm{h})$ & 0.6190 & 0.7062 & 0.6740 & 0.6360 \\
Flow rate of propane, $m_{C 3}(\mathrm{~kg} / \mathrm{h})$ & 2.847 & 2.930 & 2.649 & 2.288 \\
$\quad$ Constraints & 3.0 & 3.0 & 3.0 & 3.0 \\
$\quad$ MITA $\left({ }^{\circ} \mathrm{C}\right)$ & $\mathbf{9 2 . 0}$ & $\mathbf{9 2 . 0}$ & $\mathbf{9 2 . 0}$ & $\mathbf{9 2 . 0}$ \\
$\quad$ Liquefaction rate (\%) & $\mathbf{0 . 4 4 0 0}$ & $\mathbf{0 . 4 0 3 4}$ & $\mathbf{0 . 3 8 6 2}$ & $\mathbf{0 . 3 6 9 1}$ \\
Required specific power \\
(kWh/kg-LNG)
\end{tabular}


Table 8. Summary and comparison of the optimization results of the VS-optimized C3MR with optimized C3MR process using other well-known optimization algorithms.

\begin{tabular}{ccccc}
\hline Decision Variables & Base Case [44] & GA Optimized & PSO Optimized & VS Optimized \\
\hline MR cycle & & & & \\
Condensation pressure of $\mathrm{MR}, \mathrm{P}_{8}(\mathrm{bar})$ & 55.0 & 61.24 & 50.0 & 43.43 \\
Evaporation pressure, $\left(\mathrm{P}_{6 \mathrm{a}}, \mathrm{P}_{6 \mathrm{~b}}\right)(\mathrm{bar})$ & 3.0 & 4.55 & 5.49 & 2.75 \\
Flow rate of nitrogen, $m_{\mathrm{N} 2}(\mathrm{~kg} / \mathrm{h})$ & 0.30 & 0.2331 & 0.2519 & 0.08 \\
Flow rate of methane, $m_{\mathrm{C} 1}(\mathrm{~kg} / \mathrm{h})$ & 0.75 & 0.5764 & 0.5959 & 0.4490 \\
Flow rate of ethane, $m_{\mathrm{C} 2}(\mathrm{~kg} / \mathrm{h})$ & 0.95 & 0.9204 & 1.210 & 0.8430 \\
Flow rate of propane, $m_{\mathrm{C} 3}(\mathrm{~kg} / \mathrm{h})$ & 0.70 & 0.4989 & 0.30 & 0.5690 \\
& Propane cycle & & & 18.40 \\
Propane 1st cooling stage $\left({ }^{\circ} \mathrm{C}\right)$ & 20.00 & 19.48 & 15.54 & 0.0 \\
Propane 2nd cooling stage $\left({ }^{\circ} \mathrm{C}\right)$ & 3.5 & 1.919 & 4.50 & -16.75 \\
Propane 3rd cooling stage $\left({ }^{\circ} \mathrm{C}\right)$ & -16.0 & -15.27 & -14.70 & 3.0 \\
Constraints & & & & $\mathbf{9 2 . 0}$ \\
MITA $\left({ }^{\circ} \mathrm{C}\right)$ & 3.0 & 3.0 & 3.0 & $\mathbf{9 2 . 0}$ \\
Liquefaction rate $(\%)$ & $\mathbf{9 2 . 0}$ & $\mathbf{9 2 . 0}$ & $\mathbf{0 . 2 7 5 0}$ \\
Required specific power $(\mathbf{k W h} / \mathbf{k g}-\mathrm{LNG})$ & $\mathbf{0 . 3 6 0 2}$ & $\mathbf{0 . 2 7 7 8}$ & $\mathbf{0 . 2 7 5}$ & $\mathbf{2 3 . 5}$ \\
Relative energy saving $(\%)$ & - & $\mathbf{2 2 . 9}$ & &
\end{tabular}

\section{Exergy Destruction Analysis and Figure of Merit}

The exergy destruction of the C3MR process for all cases (base case, GA-optimized, PSO-optimized, and VS-optimized) is shown in Figure 8. Exergy analysis of propane precooling refrigeration cycles and mixed refrigerant refrigeration cycles were performed, separately (Figure 8). In the case of propane precooling section, the highest exergy destruction was found in VS-optimized air coolers and compressors, i.e., 67.3 and $73.5 \mathrm{~kJ} / \mathrm{h}$, respectively. However, in case of VS-optimized MR section of C3MR process, the exergy destruction through primary LNG exchanger was reduced by $69.5 \%$ as compared to the base case. Moreover, exergy destruction through compressors was reduced by $34 \%$ as compared to the base case. It was observed that compressors and main LNG exchanger of MR section have the highest exergy destructions, which lead to lower exergy efficiency of that section in comparison to propane precooling section.

Exergy analysis for SMR process is illustrated in Figure 9. According to Figure 9, the exergy analysis in comparison to other components was also performed. The highest exergy destruction was found in VS-optimized primary LNG exchanger, i.e., 38.5\%. In addition, in both C3MR and SMR processes, primary LNG exchanger and compressors can be further optimized by stochastic optimization algorithm (i.e., Coggins [45], modified coordinate descent, etc.) in minimization of exergy destruction.

In addition to exergy destruction minimization, Figure of Merit (FOM) plays a vital role to design efficient refrigeration and liquefaction processes. The higher value of FOM presents higher energy efficiency, and vice versa. Table 9 lists the FOM analysis of C3MR and SMR processes. Accordingly, the overall FOM of VS-optimized C3MR and SMR were calculated as $47.0 \%$ and $27.9 \%$, respectively. In case of propane precooling section, the lowest total energy was observed due to the lower range of temperature used in propane 2nd and 3rd stages of C3MR process (see Table 8). 


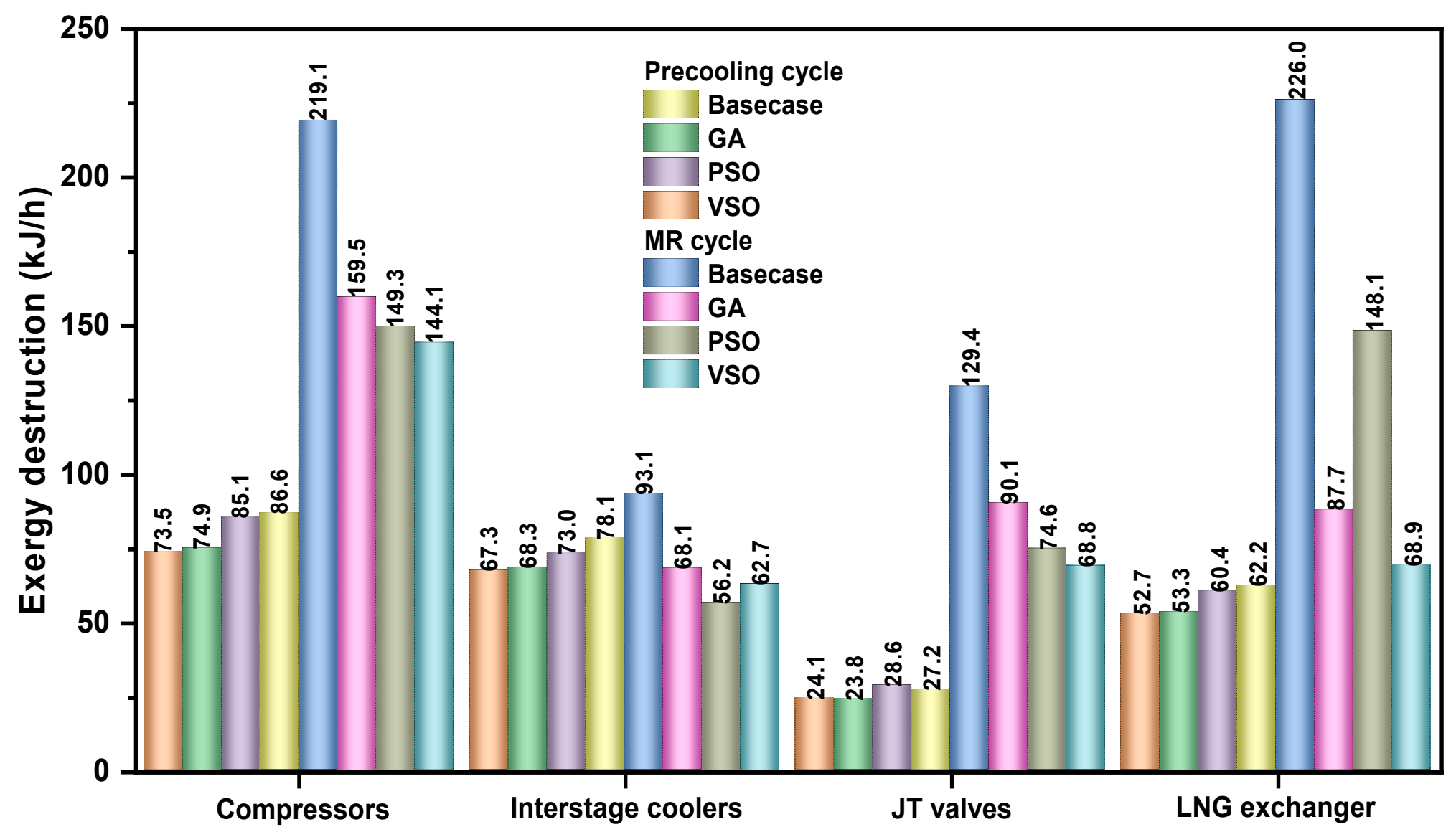

Figure 8. Exergy destruction associated with each equipment of the optimized C3MR process. 


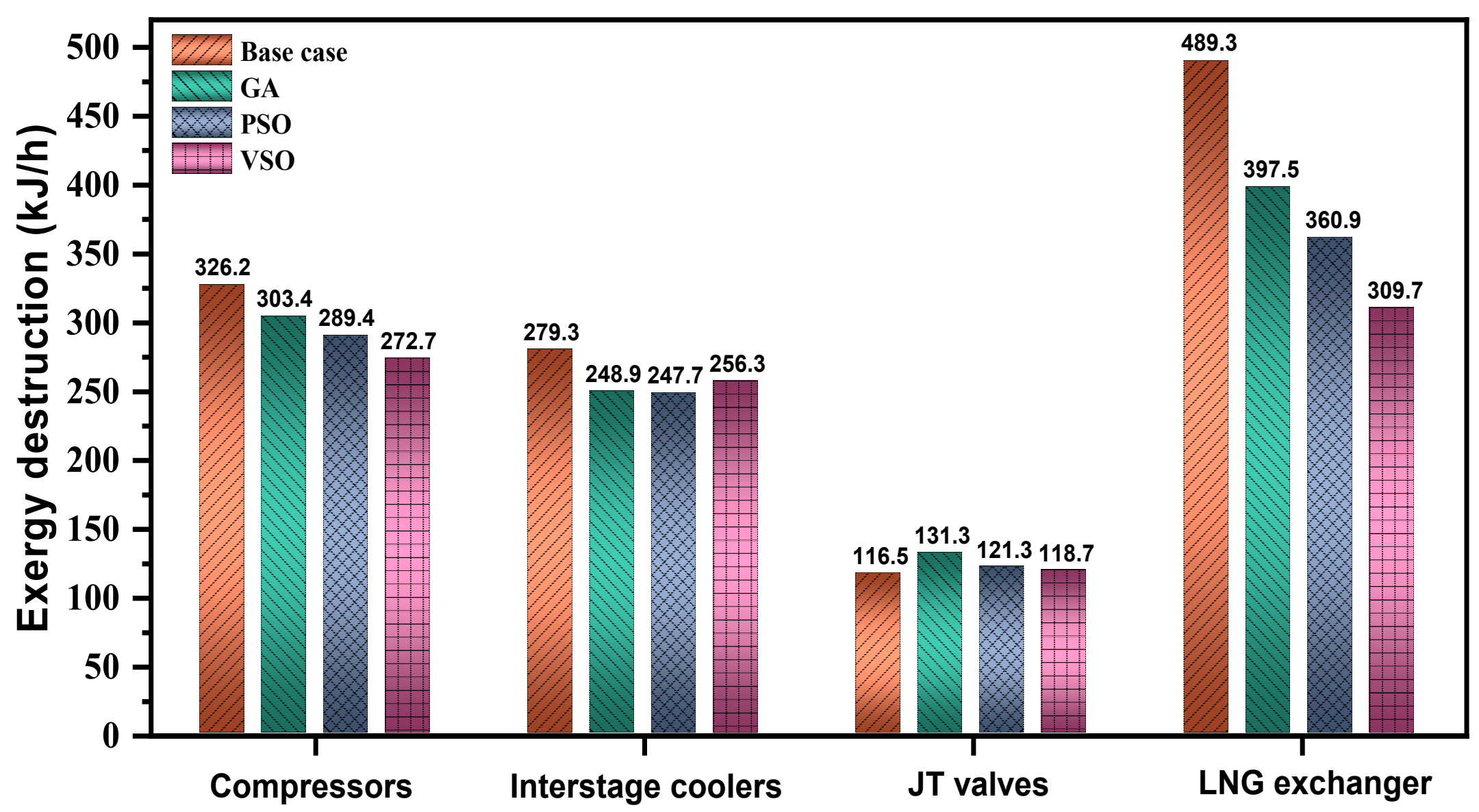

Figure 9. Exergy destruction associated with each equipment of the optimized SMR process. 
Table 9. Figure of merit (FOM) comparison analysis with base case for C3MR and SMR processes.

\begin{tabular}{|c|c|c|c|c|c|c|}
\hline \multicolumn{7}{|c|}{ FOM for C3MR Process } \\
\hline \multirow{3}{*}{ MFC Process } & \multicolumn{3}{|c|}{ Precooling Section } & \multicolumn{3}{|c|}{ MR Section } \\
\hline & Min. Required Work & Total Energy & FOM & Min. Required Work & Total Energy & FOM \\
\hline & $\mathrm{kJ} / \mathrm{h}$ & $\mathrm{kJ} / \mathrm{h}$ & $\%$ & $\mathrm{~kJ} / \mathrm{h}$ & $\mathrm{kJ} / \mathrm{h}$ & $\%$ \\
\hline Base Case & 99.69 & 356.4 & 28.08 & 356.13 & 943.2 & 37.81 \\
\hline GA_C3MR & 86.03 & 306 & 28.00 & 356.13 & 691.2 & 51.40 \\
\hline PSO_C3MR & 101.19 & 349.2 & 28.96 & 356.13 & 640.8 & 55.48 \\
\hline VSO_C3MR & 83.63 & 302.4 & 27.68 & 356.14 & 633.6 & 56.18 \\
\hline \multicolumn{7}{|c|}{ FOM for SMR Process } \\
\hline Base Case & 372.22 & 1584 & 23.50 & & & \\
\hline GA_SMR & 371.17 & 1440 & 25.56 & & & \\
\hline PSO_SMR & 371.17 & 1404 & 26.69 & & & \\
\hline VSO_SMR & 371.20 & 1332 & 27.94 & & & \\
\hline
\end{tabular}

\section{Conclusions}

This study investigated the optimization by applying a VS algorithm to improve the design of workhorse commercial SMR and C3MR cycles corresponding to the total required energy. The VS algorithm utilized the advantage of simple implementation and enhanced search by exploiting a more rigorous and sophisticated vortex pattern search via balancing the weak and strong localities in neighborhood candidate solutions. Furthermore, it was observed that its implementation for optimal search in this type of a complex LNG process was achieved via tuning only a few parameters over GA and PSO. The algorithm was extremely sophisticated such that the constraints were easily handled via augmenting into the objective function and producing results with assurance the defined temperature approach (i.e., $3{ }^{\circ} \mathrm{C}$ ) for both the SMR and C3MR processes. In the study, it was observed that the VS-optimized SMR and C3MR processes consumed 16.1\% and 27.8\% less energy, respectively, when compared to that of the base case study, respectively. Furthermore, the results obtained by the VS algorithm exhibited superior performance with low parameter adjustment when compared to those of GA and PSO. Exergy destruction analysis of the VS-optimized C3MR and SMR liquefaction processes revealed that the liquefaction process could be improved further through better compressor, intercooler, and LNG exchanger designs or further design optimization. This study does not claim the superiority of the VS algorithm for general problems but highlights its potential and possibility for finding an improved optimal solution for the particular LNG processes. It is expected that the VS algorithm is an easy and systematic approach. Hence, a future study will investigate using the approach in other MR-based LNG processes including dual MR and cascade processes.

Author Contributions: Conceptualization, M.A.Q. and M.Y.; methodology, A.N. and W.A.; software, T.H.; formal analysis, K.Q. and A.N.; investigation, T.H. and M.A.Q.; resources, J.H.; data curation, M.L.; writing-original draft preparation, M.A.Q. and J.H; writing-review and editing, A.-S.N. and K.M.; supervision, M.Y. All authors have read and agreed to the published version of the manuscript.

Funding: This work was supported by the 2020 Yeungnam University Research Grant, the Priority Research Centers Program through the National Research Foundation (NRF) of Korea funded by the Ministry of Education (2014R1A6A1031189), and by the Fundamental Research Funds for the Central Universities (No. 19CX02011A).

Conflicts of Interest: The authors declare no conflict of interest. 


\section{Nomenclature}

\section{Abbreviations}

V9

$\zeta \quad$ Haphazardly yielded variable

$\mu \quad$ Sample mean

r Radius

JT Joule-Thomson

$\mathrm{K}$ Compressor

C Cooler

Q Heat

${ }^{\circ} \mathrm{C} \quad$ Degree Celsius

$\mathrm{kg} \quad$ Kilogram

kW Kilowatt

Acronyms

C3MR Propane precooled mixed refrigerant

SMR Single mixed refrigerant

LNG Liquified natural gas

VS Vortex search

NG Natural gas

GHG Greenhouse gas

MR Mixed refrigerant

NLP Nonlinear programing

SQP Sequential quadratic programming

GA Genetic algorithm

SRQP Successive reduced quadratic programming

SCRS Sequential coordinate random search

PSO Particle swarm optimization

VSO Vortex search optimization

MFC Mixed fluid cascade

PRICO Poly refrigerant integrated cycle operations

MITA Minimum internal temperature approach

THCC Temperature heat-flow composite curve

TDCC Temperature difference composite curve

Subscripts

Xbest Best solution

o Initialization

So Standard deviation

$\mathrm{N}_{2} \quad$ Nitrogen

$\mathrm{C}_{1} \quad$ Methane

$\mathrm{C}_{2} \quad$ Ethane

$\mathrm{C}_{3} \quad$ Propane

$\mathrm{W}_{\mathrm{i}} \quad$ Compressors work

Ex $_{\text {des }} \quad$ Exergy destruction

$\mathrm{Ex}_{\text {in }} \quad$ Exergy in

Ex $_{\text {out }} \quad$ Exergy out

\section{References}

1. Chong, Z.R.; Yang, S.H.B.; Babu, P.; Linga, P.; Li, X.-S. Review of natural gas hydrates as an energy resource: Prospects and challenges. Appl. Energy 2016, 162, 1633-1652. [CrossRef]

2. ExxonMobil Corporation. The 2015 Outlook for Energy: A View to 2040. Available online: https: //corporate.exxonmobil.com/ (accessed on 3 December 2019). 
3. Rogala, Z.; Brenk, A.; Malecha, Z. Theoretical and numerical analysis of freezing risk during LNG evaporation process. Energies 2019, 12, 1426. [CrossRef]

4. Koo, J.; Oh, S.-R.; Choi, Y.-U.; Jung, J.-H.; Park, K. Optimization of an organic rankine cycle system for an LNG-powered ship. Energies 2019, 12, 1933. [CrossRef]

5. Qyyum, M.A.; Qadeer, K.; Minh, L.Q.; Haider, J.; Lee, M. Nitrogen self-recuperation expansion-based process for offshore coproduction of liquefied natural gas, liquefied petroleum gas, and pentane plus. Appl. Energy 2019, 235, 247-257. [CrossRef]

6. Eser, P.; Chokani, N.; Abhari, R. Impact of nord stream 2 and LNG on gas trade and security of supply in the European gas network of 2030. Appl. Energy 2019, 238, 816-830. [CrossRef]

7. Hönig, V.; Prochazka, P.; Obergruber, M.; Smutka, L.; Kučerová, V. Economic and technological analysis of commercial LNG production in the EU. Energies 2019, 12, 1565. [CrossRef]

8. Qyyum, M.A.; Qadeer, K.; Lee, M. Comprehensive review of the design optimization of natural gas liquefaction processes: Current status and perspectives. Ind. Eng. Chem. Res. 2018, 57, 5819-5844. [CrossRef]

9. Strantzali, E.; Aravossis, K.; Livanos, G.A.; Chrysanthopoulos, N. A novel multicriteria evaluation of small-scale LNG supply alternatives: The case of greece. Energies 2018, 11, 903. [CrossRef]

10. Qyyum, M.A.; Duong, P.L.T.; Minh, L.Q.; Lee, S.; Lee, M. Dual mixed refrigerant LNG process: Uncertainty quantification and dimensional reduction sensitivity analysis. Appl. Energy 2019, 250, 1446-1456. [CrossRef]

11. Khan, M.S.; Lee, S.; Rangaiah, G.P.; Lee, M. Knowledge based decision making method for the selection of mixed refrigerant systems for energy efficient LNG processes. Appl. Energy 2013, 111, 1018-1031. [CrossRef]

12. Khan, M.S.; Lee, M. Design optimization of single mixed refrigerant natural gas liquefaction process using the particle swarm paradigm with nonlinear constraints. Energy 2013, 49, 146-155. [CrossRef]

13. Lee, G.C.; Smith, R.; Zhu, X.X. Optimal synthesis of mixed-refrigerant systems for low-temperature processes. Ind. Eng. Chem. Res. 2002, 41, 5016-5028. [CrossRef]

14. Shah, N.M.; Hoadley, A.F.A.; Rangaiah, G.P. Inherent safety analysis of a propane precooled gas-phase liquified natural gas process. Ind. Eng. Chem. Res. 2009, 48, 4917-4927. [CrossRef]

15. Aspelund, A.; Gundersen, T.; Myklebust, J.; Nowak, M.P.; Tomasgard, A. An optimization-simulation model for a simple LNG process. Comput. Chem. Eng. 2010, 34, 1606-1617. [CrossRef]

16. Khan, M.S.; Lee, S.; Lee, M. Optimization of single mixed refrigerant natural gas liquefaction plant with nonlinear programming. Asia-Pacific J. Chem. Eng. 2012, 7, S62-S70. [CrossRef]

17. Wang, M.; Zhang, J.; Xu, Q.; Li, K. Thermodynamic-analysis-based energy consumption minimization for natural gas liquefaction. Ind. Eng. Chem. Res. 2011, 50, 12630-12640. [CrossRef]

18. Hatcher, P.; Khalilpour, R.; Abbas, A. Optimisation of LNG mixed-refrigerant processes considering operation and design objectives. Comput. Chem. Eng. 2012, 41, 123-133. [CrossRef]

19. Lee, I.; Tak, K.; Kwon, H.; Kim, J.; Ko, D.; Moon, I. Design and optimization of a pure refrigerant cycle for natural gas liquefaction with subcooling. Ind. Eng. Chem. Res. 2014, 53, 10397-10403. [CrossRef]

20. Mortazavi, A.; Alabdulkarem, A.; Hwang, Y.; Radermacher, R. Novel combined cycle configurations for propane pre-cooled mixed refrigerant (APCI) natural gas liquefaction cycle. Appl. Energy 2014, 117, 76-86. [CrossRef]

21. Lee, Y.; Huh, C.; Lee, W.B. Inherently safer process design of natural gas liquefaction processes through multiobjective optimization-Part I. with inherent safety indexes. Ind. Eng. Chem. Res. 2019, 58, 4186-4198. [CrossRef]

22. Lee, I.; Moon, I. strategies for process and size selection of natural gas liquefaction processes: Specific profit portfolio approach by economic based optimization. Ind. Eng. Chem. Res. 2018, 57, 5845-5857. [CrossRef]

23. Tsay, C.; Baldea, M. Scenario-free optimal design under uncertainty of the PRICO natural gas liquefaction process. Ind. Eng. Chem. Res. 2018, 57, 5868-5880. [CrossRef]

24. Pattison, R.C.; Baldea, M. Multistream heat exchangers: Equation-oriented modeling and flowsheet optimization. AIChE J. 2015, 61, 1856-1866. [CrossRef]

25. Tsay, C.; Pattison, R.C.; Baldea, M. Equation-oriented simulation and optimization of process flowsheets incorporating detailed spiral-wound multistream heat exchanger models. AIChE J. 2017, 63, 3778-3789. [CrossRef]

26. Vikse, M.; Watson, H.A.J.; Gundersen, T.; Barton, P.I. Versatile simulation method for complex single mixed refrigerant natural gas liquefaction processes. Ind. Eng. Chem. Res. 2018, 57, 5881-5894. [CrossRef] 
27. Ali, W.; Qyyum, M.A.; Khan, M.S.; Duong, P.L.T.; Lee, M. Knowledge-inspired operational reliability for optimal LNG production at the offshore site. Appl. Therm. Eng. 2019, 150, 19-29. [CrossRef]

28. Zhu, J.; Zhang, W.; Li, Y.; Ji, P.; Wang, W. Experimental study of flow distribution in plate-fin heat exchanger and its influence on natural gas liquefaction performance. Appl. Therm. Eng. 2019, 155, 398-417. [CrossRef]

29. Xu, X.; Liu, J.; Jiang, C.; Cao, L. The correlation between mixed refrigerant composition and ambient conditions in the PRICO LNG process. Appl. Energy 2013, 102, 1127-1136. [CrossRef]

30. Qadeer, K.; Qyyum, M.A.; Lee, M. Krill-herd-based investigation for energy saving opportunities in offshore liquefied natural gas processes. Ind. Eng. Chem. Res. 2018, 57, 14162-14172. [CrossRef]

31. Doğan, B.; Ölmez, T. A new metaheuristic for numerical function optimization: Vortex search algorithm. Inf. Sci. 2015, 293, 125-145. [CrossRef]

32. Ali, W.; Qyyum, M.A.; Qadeer, K.; Lee, M. Energy optimization for single mixed refrigerant natural gas liquefaction process using the metaheuristic vortex search algorithm. Appl. Therm. Eng. 2018, 129, 782-791. [CrossRef]

33. Chaniago, Y.D.; Qyyum, M.A.; Andika, R.; Ali, W.; Qadeer, K.; Lee, M. Self-recuperative high temperature co-electrolysis-based methanol production with vortex search-based exergy efficiency enhancement. J. Clean. Prod. 2019, 239, 118029. [CrossRef]

34. Doğan, B.; Ölmez, T. Vortex search algorithm for the analog active filter component selection problem. AEU Int. J. Electron. Commun. 2015, 69, 1243-1253. [CrossRef]

35. Doğan, B.; Yüksel, A. Analog filter group delay optimization using the vortex search algorithm. In Proceedings of the 23nd Signal Processing and Communications Applications Conference (SIU), Malatya, Turkey, 16-19 May 2015; pp. 288-291.

36. He, T.; Karimi, I.A.; Ju, Y. Review on the design and optimization of natural gas liquefaction processes for onshore and offshore applications. Chem. Eng. Res. Des. 2018, 132, 89-114. [CrossRef]

37. Rehman, A.; Qyyum, M.A.; Qadeer, K.; Zakir, F.; Ding, Y.; Lee, M.; Wang, L. Integrated biomethane liquefaction using exergy from the discharging end of a liquid air energy storage system. Appl. Energy 2020, 260, 114260. [CrossRef]

38. Khan, M.S.; Karimi, I.A.; Wood, D.A. Retrospective and future perspective of natural gas liquefaction and optimization technologies contributing to efficient LNG supply: A review. J. Nat. Gas Sci. Eng. 2017, 45, 165-188. [CrossRef]

39. Mortazavi, A.; Somers, C.; Alabdulkarem, A.; Hwang, Y.; Radermacher, R. Enhancement of APCI cycle efficiency with absorption chillers. Energy 2010, 35, 3877-3882. [CrossRef]

40. Veatch, B. LNG Engineering \& Plant Construction. Available online: https://www.bv.com/industries/oil-gas/ liquefied-natural-gas-lng (accessed on 23 March 2020).

41. Qyyum, M.A.; Qadeer, K.; Lee, S.; Lee, M. Innovative propane-nitrogen two-phase expander refrigeration cycle for energy-efficient and low-global warming potential LNG production. Appl. Therm. Eng. 2018, 139, 157-165. [CrossRef]

42. Venkatarathnam, G.; Timmerhaus, K. Cryogenic Mixed Refrigerant Processes; Springer: Berlin/Heidelberg, Germany, 2008; Volume 100.

43. Ding, H.; Sun, H.; He, M. Optimisation of expansion liquefaction processes using mixed refrigerant $\mathrm{N}_{2}-\mathrm{CH}_{4}$. Appl. Therm. Eng. 2016, 93, 1053-1060. [CrossRef]

44. Khan, M.S.; Karimi, I.A.; Bahadori, A.; Lee, M. Sequential coordinate random search for optimal operation of LNG (liquefied natural gas) plant. Energy 2015, 89, 757-767. [CrossRef]

45. Nawaz, A.; Qyyum, M.A.; Qadeer, K.; Khan, M.S.; Ahmad, A.; Lee, S.; Lee, M. Optimization of mixed fluid cascade LNG process using a multivariate Coggins step-up approach: Overall compression power reduction and exergy loss analysis. Int. J. Refrig. 2019, 104, 189-200. [CrossRef]

(C) 2020 by the authors. Licensee MDPI, Basel, Switzerland. This article is an open access article distributed under the terms and conditions of the Creative Commons Attribution (CC BY) license (http://creativecommons.org/licenses/by/4.0/). 